J. Austral. Math. Soc. 19 (Series A), (1975), 62-73.

\title{
CAUCHY COMPLETION OF ABELIAN TIGHT RIESZ GROUPS
}

\author{
B. F. SHERMAN \\ (Received 13 March 1973, Revised 28 June 1973) \\ Communicated by G. E. Wall
}

\section{Introduction}

This paper concerns the completions of partially ordered groups introduced by Fuchs (1965a) and the author (to appear); the p.o. groups under consideration are, generally, abelian tight Riesz groups, and so, throughout, the word "group" will refer to an abelian group.

In section 3 we meet the cornerstone of the work, the central product theorem, by means of which we can interpret the Cauchy completion of a tight Riesz group in terms of the completion of any of its $o$-ideals; one particularly important case arises when the group has a minimal $o$-ideal. Such a minimal $o$-ideal is $o$-simple, and in section 6 the completion of an isolated $o$-simple tight Riesz group is shown to be a tight Riesz real vector space. If the group has no minimal ideal, then we can consider the possibility that every interval around the zero contains an $o$ ideal (e.g., an infinite lexicographic product of fully ordered groups); in such a case it is shown in section 4 that the completion is $o$-isomorphic to the projective limit of the factor groups of the $o$-ideals. If this possibility does not hold, then we have some $o$-ideal in which there are no bounded $o$-ideals, and a strong unit; so, by virtue of the central product theorem, in section 5 we look at isolated tight Riesz groups which have a strong unit and no bounded subgroups. These are found to be $o$-isomorphic to groups of bounded real functions and, further, if the associated order of the group is para-archimedean, then the completion is also $o$-isomorphic to a group of bounded real functions, with domain the same as for those functions representing the original group.

Several unanswered questions arise from the work: In section 5, is it possible to characterise all positive elements in terms of the domain of the functions? Are there any tight Riesz groups without pseudozeros having a bounded subgroup but no bounded $o$-ideal? In section 6 , are all $o$-simple abelian tight Riesz groups isolated? Section 7 provides some examples, used both to illustrate theorems and to contradict certain hypotheses which presented themselves. 


\section{Preliminaries}

For those terms and concepts in common use the reader is referred to Fuchs (1963); the only terminological variations are:

(i) the additive notation $(A,+)$ is used;

(ii) the positive cone $P=P(A)=\{x \in A \mid x>0\}$ excludes 0 ;

(iii) an abelian p.o. group is $o$-simple if it has no non-trivial $o$-ideals; or, equivalently, if for any $a, b \in A$ with $a>0$ there is an integer $n$ such that $n a>b$;

(iv) "Completely integrally closed" becomes "para-archimedean", as in Ribenboim (1969).

For Riesz groups, o-monomorphisms, denseness, pseudopositives and pseudozeros (cf. pseudoidentities) the reader is referred to Fuchs (1965), whilst the projective (or inverse) limit is described in Schaefer (1966) and Graetzer (1968).

The tight Riesz $(T R(2,2))$ property is: if $a, b<d, e$ in $(A, \geqq)$, then there is a $c \in A$ such that $a, b<c<d, e$; and the $T R(1,2)$ property is: if $b<d, e$ in $(A, \geqq)$, then there is a $c \in A$ such that $b<c<d$,e. Tight Riesz groups and $T R(1,2)$ groups are then directed p.o. groups with the respective properties, whilst tight Riesz vector spaces are tight Riesz g roups which are also real vector spaces, and such that multiplication by a positive real preserves order.

LEMMA 2.1. The intersection of two non-zero o-ideals of a tight Riesz group is also a non-zero o-ideal.

Proof: Let $(A, \geqq)$ be the tight Riesz group, and $B$ and $C$ the non-zero $o$-ideals. By Fuchs ((1965), proposition 5.4) $B \cap C$ is an $o$-ideal; and taking $b>0$ in $B$ and $c>0$ in $C$ we find, by the tight Riesz property, an $a \in A$ such that $b, c>a>0$; so, by convexity, $a \in B \cap C$, and so $B \cap C$ is non-zero.

COROLlary 2.2. Any minimal non-zero o-ideal of a tight Riesz group is the unique minimum non-zero o-ideal of the group.

Proof. Let $M$ be a minimal non-zero $o$-ideal of $(A, \geqq)$, and $C$ any other nonzero $o$-ideal; then $M \cap C=M$, by the minimality of $M$, and so $M \subseteq C$.

Throughout this paper any p.o. group will be considered as having its open interval topology $\mathscr{U}$, and any reference to topological properties or concepts of a p.o. group will be to this topology.

For further properties of tight Riesz groups, particularly with respect to the open interval tolopogy thereon, the reader is referred to Loy and Miller (1972); we note especially that a tight Riesz group is a topological group. We shall call the pre-ordered group $(A, \succcurlyeq)$, derived from $(A, \geqq)$ in Loy and Miller (1972), the associated order of $(A, \geqq)$; in effect, $x \geqslant 0$ if and only if $u>x \Rightarrow u>0$. Note that also that $x>0 \Rightarrow x \geqslant 0$, and $x \geqslant y>0 \Rightarrow x>0$. $(A, \geqslant)$ is a p.o. group if and only if $(A, \geqq)$ has no pseudozeros; i.e. if only and if $(A, \mathscr{U})$ is Hausdorff.

If $(A, \mathscr{U})$ is a topological group we may form the Cauchy completion $\left(A^{*}, \geqq\right)$ 
(cf. Sherman (to appear)) by taking $A^{*}$ to be the group of round Cauchy $O$-filters on $\mathscr{U}$, and order $A^{*}$ by taking $P\left(A^{*}\right)=\left\{\mathscr{F} \in A^{*} \mid P(A) \in \mathscr{F}\right\}$. An $O$-filter $\mathscr{F}$ is a non-empty set of non-empty open subsets of $A$, which is closed under intersection, and includes all open supersets of its elements; it is Cauchy if, for any open set $V$ containing 0 , there is an $F \in \mathscr{F}$ such that $F-F \subseteq V$; and round if for any $H \in \mathscr{F}$ there is a $G \in \mathscr{F}$ and an open set $U$ containing 0 such that $G+U \subseteq H$. For any $a \in A$ the neighbourhoods $O$-filter $V(a)$ is the set of open sets containing $a$; the map $\theta: A \rightarrow A^{*}, a \theta=V(a)$ is called the natural embedding of $A$ in $A^{*}$; it is an $o$-homomorphism, and, if $(A, \geqq)$ has no pseudozeros, is an $o$-monomorphism. If $(A, \geqq)$ is a tight Riesz group, then so is $\left(A^{*}, \geqq\right)$ and $A \theta$ is then a dense subgroup of $\left(A^{*}, \geqq\right)$.

The Fuchs completion of a tight Riesz group $(A, \geqq)$ is the same group, $A^{*}$, with the ordering given by: $\mathscr{F} \succcurlyeq \mathscr{V}(0)$ if and only if for all $F \in \mathscr{F}, P(A) \cap F$ is non empty. (cf. Sherman (to appear), and Fuchs (1965a), page 193). Then the Fuchs completion $\left(A^{*}, \succcurlyeq\right)$ in fact the associated order of the Cauchy completion $\left(A^{*}, \geqq\right)$ (Sherman (to appear), theorem 5 ). An approximation antilattice $(A, \geqq)$ is a tight Riesz group such that for any $a, b$ and $u \in A$, where $u>0$, there is a $c \in A$ such that

(i) $c<a, b$; and

(ii) if $x<a, b$ in $A$, then $x<c+u$.

Fuchs (1965a) has shown that the Fuchs completion of an approximation antilattice is lattice-ordered, whilst the author (to appear) has shown that approximation antilattices are the dense subgroups of tight Riesz groups with latticeordered associated order.

The central product (Reilly, (1972)) of two p.o. (abelian) groups $(A, \geqq)$ and $(B, \geqq)$ amalgamating two $o$-isomorphic subgroups $C \subseteq A$ and $C \psi \subseteq B$ (where $\psi$ is the $o$-isomorphism) is the factor group $(A \times B) / N$, where $N=\{(c,-c \psi) \mid c \in C\}$ $\subseteq A \times B$. Note that $A$ is $o$-isomorphic to $(A \times\{0\})+N, B o$-isomorphic to $(\{0\} \times B)+N$, and $C o$-isomorphic to $(C \times\{0\})+N=(\{0\} \times C \psi)+N$.

\section{Structure of the Completion}

In this section the connections between the $o$-ideals of the p.o. group and those of its completion are investigated. We shall suppose $(B, \geqq)$ to be an $o$-ideal of $(A, \geqq)$, and define the subset $B^{\prime}$ of $A^{*}$ to be $\left\{\mathscr{F} \in A^{*} \mid \mathscr{V}(b)<\mathscr{F}<\mathscr{V}(c)\right.$ for some $b, c \in B\} . B^{\prime}$ is clearly an $o$-ideal of $A^{*}$. Similarly, if $(C, \geqq)$ is an $o$-ideal of $A^{*}$, then $(C \cap A \theta) \theta^{-1}$, where $\theta$ is the natural embedding, will be an $o$-ideal of $A$; and $\left(B^{\prime} \cap A \theta\right) \theta^{-1}=B$, whilst $\left[(C \cap A \theta) \theta^{-1}\right]^{\prime}=C$.

To distinguish between $A$ and $B$ I shall use $(a, b)_{A}=\{x \in A \mid a<x<b\}$, and $(a, b)_{B}=\{x \in B \mid a<x<b\} ; \mathscr{V}_{A}(a)$ will denote the neighbourhoods $O$-filter of $a$ in $A^{*}$, whilst $\mathscr{V}_{B}(a)$ will be that in $B^{*}$.

LeMMA 3.1. ( $\left.B^{\prime}, \geqq\right)$ is a o-isomorphic to $\left(B^{*}, \geqq\right)$. 
Proof: The required $o$-isomorphism is $\phi$, where, for any $\mathscr{F} \in B^{\prime}, \mathscr{F} \phi=\{G \mid G$ $=F \cap B$ for some $F \in \mathscr{F}\}$; that $\mathscr{F} \phi \in B^{*}$ follows from the corresponding properties of $\mathscr{F}$, except that we need the fact that $\mathscr{F} \in B^{\prime}$ to show that each $G \in \mathscr{F} \phi$ is non-empty. Moreover, $\mathscr{F} \phi+\mathscr{G} \phi=(\mathscr{F}+\mathscr{G}) \phi$ follows readily from the definition of addition of $O$-filters; whilst the fact that $P(A) \cap B=P(B)$ shows that $\mathscr{F} \phi>\mathscr{V}_{B}(0)$ if and only if $\mathscr{F}>\mathscr{V}_{A}(0) . \phi^{-1}$ is given by: if $\mathscr{G} \in \mathscr{B}{ }^{*}$, then $\mathscr{G} \phi^{-1}$ is the $O$-filter with $\left\{(a, b)_{A} \mid a, b \in B\right.$ and $\left.(a, b)_{B} \in \mathscr{G}\right\}$ as a base. That $\mathscr{G} \phi^{-1}$ is both round and Cauchy follows from those properties of $\mathscr{G}$; whilst $\mathscr{F} \phi \phi^{-1}=\mathscr{F}$, and $\mathscr{G} \phi^{-1} \phi=\mathscr{G}$ are easily verified.

In fact $\mathscr{V}_{A}(b) \phi=\mathscr{V}_{B}(b)$ for each $b \in B$.

LeMMA $3.2\left(A^{*} / B^{\prime}, \geqq\right)$. is o-isomorphic to $(A / B, \geqq)$.

PRoOF: The required $o$-isomorphism is $\rho: A / B \rightarrow A^{*} / B^{\prime}$, where $(x+B) \rho$ $=\mathscr{V}_{A}(x)+B^{\prime}$. Clearly $\rho$ is a one to one o-isomorphism. - is also onto; for if take $\mathscr{F}+B^{\prime} \in A^{*} / B^{\prime}$, then we can take $\mathscr{W}>\mathscr{V}_{A}(o)$ in $B^{\prime}$, so that $\mathscr{F}+\mathscr{W} \in \mathscr{F}$ $+B^{\prime} ;$ as $A \theta$ is dense in $A^{*}$, we can find $x \in A$ such that $\mathscr{F}+\mathscr{W}>\mathscr{V}_{A}(x)>\mathscr{F}$; and hence $\mathscr{V}_{A}(x) \in \mathscr{F}+B^{\prime}$; i.e. $\mathscr{V}_{A}(x)+B^{\prime}=\mathscr{F}+B^{\prime}$.

This indicates a marked similarity in the structures of $A$ and $A^{*}$; in fact we find

Theorem 3.3 (Central Product Theorem) If $(B, \geqq)$ is an o-ideal of $(A, \geqq)$, an abelian tight Riesz group without pseudozeros, then the completion $\left(A^{*}, \geqq\right)$ is o-isomorphic to the central product of $(A, \geqq)$ and $\left(B^{*}, \geqq\right)$ (the completion of $(B, \geqq))$ amalgamating $B$ and its image $B \psi$, where $\psi$ is the natural embedding of $B$ in $B^{*}$.

ProOf: Let $D$ be the group $\left\{\left(b, \mathscr{V}_{B}(-b)\right) \mid b \in B\right\}$; then the central product is $\left(A \times B^{*}\right) / D$. Then the required $o$-isomorphism is $\tau:\left(A \times B^{*}\right) / D \rightarrow A^{*}$, where $((a, \mathscr{G})+D) \tau=\mathscr{V}_{A}(a)+\mathscr{G} \phi^{-1}$, where $\phi: B^{\prime} \rightarrow B^{*}$ is the same as in Lemma 3.1.

Then $\tau$ is well defined; for, given $b \in A$ and $\mathscr{F} \in B^{*}$ such that $(b, \mathscr{F})+$ $D=(a, \mathscr{G})+D$, we know that $(b-a, \mathscr{F}-\mathscr{G}) \in D$. So $\mathscr{F}-\mathscr{G}=\mathscr{V}_{B}(a-b)$, thus giving $\mathscr{F} \phi^{-1}-\mathscr{G} \phi^{-1}=\mathscr{V}_{A}(a-b)=\mathscr{V}_{A}(a)-\mathscr{V}_{A}(b)$, and hence $\mathscr{V}_{A}(a)+$ $\mathscr{G} \phi^{-1}=\mathscr{V}_{A}(b)+\mathscr{F} \phi^{-1}$. Further, as in lemma 3.2, we can represent any $\mathscr{F} \in A^{*}$ in the form $\mathscr{V}_{A}(x)+\mathscr{H}$, where $x \in A$ and $\mathscr{H} \in B^{\prime}$, and hence also in the form $\mathscr{V}_{A}(x)+\mathscr{G} \phi^{-1}$, where $\mathscr{G} \in B^{*}$. One-to-one-ness follows by a reversal of the above argument, and so $\tau$ is an isomorphism.

If $a>0$ and $\mathscr{G}>\mathscr{V}_{B}(0)$, then $\mathscr{V}_{A}(a)+\mathscr{G} \phi^{-1}>\mathscr{V}_{A}(0)$; whilst if $\mathscr{F}>\mathscr{V}_{A}(0)$ in $A^{*}$, we can find $\mathscr{W} \in B^{\prime}$ such that $\mathscr{F}>\mathscr{W}>\mathscr{V}_{A}(0)$ (as $\left(A^{*}, \geqq\right)$ is a tight Riesz group) and hence $\mathscr{F}>\mathscr{F}-\mathscr{W}>\mathscr{V}_{A}(0)$. Thus there is an $a \in A$ such that $\mathscr{F}$ $>\mathscr{V}_{A}(a)>\mathscr{F}-\mathscr{W}>\mathscr{V}_{A}(0)$; so $\mathscr{F}-\mathscr{V}_{A}(a) \in B^{\prime}$, and so $\mathscr{G}=\left(\mathscr{F}-\mathscr{V}_{A}(a)\right) \phi$ $>\mathscr{V}_{B}(0), a>0$, and $\mathscr{F}=((a, \mathscr{G})+D) \tau$. Thus $\tau$ is the required $o$-isomorphism. 


\section{The Completion as a Projective Limit}

Under certain circumstances the completion of a tight Riesz group can be considered as a projective limit of the factor groups of its $o$-ideals.

The condition we require is

$\left.{ }^{*}\right)(A, \geqq)$ is a tight Riesz group, without pseudozeros, such that every open interval around 0 contains some non-zero $o$-ideal.

A fully ordered group with no minimal non-zero $o$-ideal is an example of such a group. Note that if $(A, \geqq)$ satisfies $\left(^{*}\right)$, then so does its completion $\left(A^{*}, \geqq\right)$.

Let $(\mathscr{B}, \geqq)$ and $(\mathscr{C}, \geqq)$ be the sets of non-zero $o$-ideals of $A$ and $A^{*}$ respectively, each partially ordered by reverse inclusion. From lemma 2.1 , we have that, if $B$ and $D \in \mathscr{B}$, then so does $B \cap D$; and by reverse inclusion we have $B \cap D \geqq B$, $D$. Hence $(\mathscr{B}, \geqq)$ is directed, and, similarly, so is $(\mathscr{C}, \geqq)$.

We recall that $\psi: \mathscr{B} \rightarrow \mathscr{C}, B \psi=B^{\prime}$ is a bijection (cf. $\S 3$ ); it is clear that $\psi$ preserves the partial orders. This means that the projective limit $\left(A^{P}, \geqq\right)$, taken over $(\mathscr{B}, \geqq)$ as an index set, of the factor groups of the non-zero $o$-ideals of $(A, \geqq)$, is $o$-isomorphic to the equivalent limit $\left(\left(A^{*}\right)^{P}, \geqq\right)$ for $\left(A^{*}, \geqq\right)$; for the factor groups are $o$-isomorphic (lemma 3.2.), and the index sets interchangeable.

Lemma 4.1. If $(A, \geqq)$ satisfies $\left({ }^{*}\right)$, then the map $\sigma: A \rightarrow A^{P}$, a $\sigma=$ $(\cdots, a+B, \cdots)$ is an o-monomorphism.

Proof: This will be the case as long as 0 is the only element belonging to every non-zero $o$-ideal. Take any non-zero $g \in A$; then there is an $h>0$ in $A$ such that either $h \geq g$ or $g-h$, because there are no pesudozeros. Let $B$ be a non-zero $o$-ideal within $(-h, h)-B$ exists by $\left({ }^{*}\right)-$ and then $g \notin B$.

THeOREM 4.2. If $(A, \geqq)$ satisfies $(*)$, then $\left(A^{P}, \geqq\right)$ is o-isomorphic to $\left(A^{*}, \geqq\right)$.

Proof: From the discussion preceding lemma 4.1, it is clear that we need only produce an $o$-isomorphism from $\left(A^{*}, \geqq\right)$ to $\left(\left(A^{*}\right)^{P}, \geqq\right)$; and, by applying the lemma to $\left(A^{*}, \geqq\right)$, we see that $\sigma^{*}: A^{*} \rightarrow\left(A^{*}\right)^{P}$ is an $o$-monomorphism. So we need only show that $\sigma^{*}$ is epimorphic.

Take $\mathbf{f}=\left(\cdots, \mathscr{F}_{c}+C, \cdots\right) \in\left(A^{*}\right)^{P} ;$ form $\mathfrak{F}=\left\{\mathbf{F} \in U^{*} \mid \mathscr{F}_{C}+C \subseteq \mathbf{F}\right.$ for some $C \in \mathscr{C}\}$. Then $\mathfrak{F}$ is a round Cauchy $O$-filter on $\left(A^{*}, \geqq\right)$; for:

(i) $\mathfrak{F}$ is a filter; if $\mathbf{N}, \mathbf{G} \in \mathfrak{F}$, then there are $C, D \in \mathscr{C}$ such that $\mathscr{F}_{C}+C \subseteq \mathbf{H}$, and $\mathscr{F}_{D}+D \subseteq \mathbf{G}$; but $\mathscr{F}_{C}+C=\mathscr{F}_{C \cap D}+C$, and $\mathscr{F}_{D}+D=\mathscr{F}_{C \cap D}+D$, by the definition of the projective limit. Thus $\mathscr{F}_{C \cap D}+C \cap D \subseteq \mathbf{H} \cap \mathbf{G}$.

(ii) $\mathfrak{F}$ is Cauchy; for, given an open set $\mathbf{V}$ around $\mathscr{V}(0)$, we have an $o$-ideal $C$ within $\mathbf{V}$. Then $\left(\mathscr{F}_{c}+C\right)-\left(\mathscr{F}_{C}+C\right)=C$.

(iii) $\mathfrak{F}$ is round; for, given $\mathbf{F} \in \mathfrak{F}$, we have an $o$-ideal $C$ such that $\left(\mathscr{F}_{C}+C\right)$ $+C=\mathscr{F}_{C}+C \subseteq \mathbf{F}$.

But $A^{*}$ is complete, so that $\mathfrak{F}$ must converge to a limit $\mathscr{F} \in A^{*}$; so $\mathfrak{F}=\mathfrak{V}(\mathscr{F})$. 
Hence, for each $C \in \mathscr{C}, \mathscr{F}_{C}+C \in \mathscr{F}$, and so $\mathscr{F}_{E} \mathscr{F}_{C}+C$; i.e., $\mathscr{F}_{C}+C=\mathscr{F}+C$ for each $C \in \mathscr{C}$. Thus $\mathbf{f}=(\cdots, \mathscr{F}+C, \cdots)=\mathscr{F} \sigma^{*}$.

\section{Real Valued Functions}

Consider a group of bounded real functions on a set $\mathscr{T}$, including the function $e(T)=1$ for all $T \in \mathscr{T}$. If $B$ has a partial order $\geqq$ satisfiying the condition:

if $f \geqq 0$ in $B$, then $f(T) \geqq 0$ for all $T \in \mathscr{T}$,

then $\geqq$ will be an $\alpha$-order if the following holds:

$(\alpha)$ if $f(T)>0$ for all $T \in \mathscr{T}$, then $f>0$ in $B$.

If instead we have the condition

$(\beta)$ if $f(T) \geqq \varepsilon>0$ for all $T \in \mathscr{T}$, then $f>0$;

then we call $\geqq$ a $\beta$-order. $B$ will then be called an $\alpha$-ordered or $\beta$-ordered function group respectively. Note that an $\alpha$-ordered function group is also a $\beta$-ordered one, but the converse need not hold.

THEOREM 5.1. An isolated abelian p.o. group $(A, \geqq)$ is o-isomorphic to an $\alpha$-ordered (or $\beta$-ordered) function group if and only if it is archimedean, has a strong unit, and no pseudozeros.

Suppose $(A, \geqq)$ is abelian, isolated, and archimedean, with strong unit and no pseudozeros. The proof utilises a theorem of Lorenzen (1939), and Dieudonné ((1941) p. 135), and proceeds as in Ribenboim ((1959), p. 75), with a minor variation to encompass the extra conditions. Basically, our index set $\mathscr{T}$ is the set of full orderes $T$ of $A$ which contain our positive cone $P$; in the theorem of Lorenzen and Dieudonne it is shown that $\bigcap\{T \in \mathscr{T}\}=P$. For each $T \in \mathscr{T}$ we form the $o$-ideal $C_{T}$ (in the ordering $T$ gives $A$ ) maximal with respect to not containing $e$; we then consider $A_{T}$, the factor group of $A$ over $C_{T} . A_{T}$ is fully ordered and archimedian, and hence $o$-isomorphic to a subgroup of the reals; in fact, as $e+C_{T}$ must always be positive in $A_{T}$, we can arrange that its image in $R$ is 1 by multiplying every image by a suitable factor. If we call the ensuing $o$-isomorphism $\phi_{T}$ and the image of $A_{T}$ in $R$ under this $R_{T}$, then the $o$-isomorphism we seek is $\phi: A \rightarrow \pi\left(R_{T} \mid T \in \mathscr{T}\right), a \phi=\left(\cdots,\left(a+C_{T}\right) \phi_{T}, \cdots\right)$.

At this point we depart from Ribenboim's proof. To show that $\phi$ is an isomorphism, we need only show that it is one-to-one, as it is clearly a homomorphism. Suppose $g \phi=(\cdots, 0, \cdots)$ for some $g \in A$; then for each $T \in \mathscr{T}$ and each integer $n$, either positive or negative, we have $n g \in C_{T}$. As $T$ is a full order on $A$, and $e+C_{T}>C_{T}$, we must have $e-n g \in T$ for each $T$; i.e., $e-n g \in \cap(T \in \mathscr{T})=P$. Hence $e>n g$ for each integer $n$; i.e., $\{n g \mid n$ an integer $\}$ is a subgroup bounded by $e$, and so is the zero subgroup; thus $g=0$. The mapping $\phi$ becomes an $o$-isomorphism if we use the induced ordering; thus it remains to show only that this obeys the conditions given - if $a>0$ in $A$, then $a+C_{T} \geqq C_{T}$ for each $T \in \mathscr{T}$, and so $\left(a+C_{T}\right) \phi_{T} \geqq 0$ for each $T \in \mathscr{T}$; whilst if $\left(a+C_{T}\right) \phi_{T} \geqq \varepsilon>0$ for 
each $T \in \mathscr{T}$, then $\left(a+C_{T}\right) \phi_{T}>0$ and so $a+C_{T}>C_{T}$ for each $T$; thus $a \in T$ for each $T \in \mathscr{T}$, and hence $a \in \bigcap\{T \in \mathscr{T}\}=P$, i.e., $a>0$. So the function group is $\beta$-ordered, and $\alpha$-ordered.

The converse follows easily.

It will be noted that the positive cone for the group of bounded real functions is not defined, but merely restricted, by the conditions given. It is found to be helpful if we require of our positive cone that any element which dominates pointwise a positive element should itself be positive; i.e., if $f(T) \geqq g(T)$ for all $T \in \mathscr{T}$ and $g>0$, then $f>0$.

An ordering with this property shall be called proper.

LEMMA 5.2. If $(A, \geqq)$ is an $\alpha$-or $\beta$-ordered function group, then the order is proper if only and if the associated order $\mathrm{W}$ is para-archimedean.

PrOOF: First, suppose the order is proper; then clearly if $f(T) \geqq 0$ for each $T \in \mathscr{T}$, then $f \succcurlyeq 0$; and if $f(T)<0$ for some $T \in \mathscr{T}$, then there is some positive integer $n$ such that $n f(T)<-1$; thus $n f(T)+e(T)<0$, so that $n f+e$ and hence $n f \geq 0$, and thus $f \geq 0$. Therefore the associated ordering is given by: $f \geqslant 0$ whenever $f(T) \geqq 0$ for each $T \in \mathscr{T}$; this is clearly para-archimedean.

Secondly, suppose the associated ordering is para-archimedean; take any $f \in A$ such that $f(T) \geqq 0$ for each $T \in \mathscr{T}$. Then $n f(T) \geqq 0>-1$ for each $T \in \mathscr{T}$ and each positive integer $n$, and so $n f>-e$ for each $n$; thus $n f \geqslant-e$ for each $n$, and therefore $f \geqslant 0$. Hence if $f(T) \geqq g(T)$ for all $T \in \mathscr{T}$, and $g>0$, then $f \geqslant g$ $>0$ and so $f>0$.

Note that if a $\beta$-order $\geqq$ is proper then condition $(\beta)$ becomes redundant, because $A$ is isolated and $e>0$.

THEOREM 5.3. If an abelian tight Riesz group $(A, \geqq)$ is o-isomorphic to a properly $\beta$-ordered function group on a set $\mathscr{T}$, then so is its completion $\left(A^{*}, \geqq\right)$. If $\phi$ and $\psi$ are the respective o-isomorphisms, $\theta$ the natural embedding of $A$ into $A^{*}$, and $i$ the inclusion map between the function groups, then $\psi \theta=i \phi$.

Proof: For any $T \in \mathscr{T}$ and any $f \in A$ define $f(T)$ to be the value taken at $T$ by $f \phi$ in the function group; then for any $\mathscr{F} \in A^{*}$ we form the real number $\mathscr{F}(T)$ as follows:-

As $(A, \geqq)$ is a tight Riesz group, for any positive integer $n$ we can find $t_{n} \in A$ such that $0<2 n t_{n}<e$, where $e(T)=1$ for all $T \in \mathscr{T}$ (cf. Loy and Miller(1972), page 236). We then define $h_{n} \in A$ recursively by the rule $0<h_{n}<t_{n}, h_{n-1}$.

Next, for each positive $n$, we take $F_{n} \in \mathscr{F}$ such that $F_{n}-F_{n} \subseteq\left(-h_{n}, h_{n}\right)$ (as $\mathscr{F}$ is Cauchy), and then choose $f_{n} \in F_{n} \cap F_{n-1} \cap \cdots \cap F_{1}$. Now $\left\{f_{n}(T)\right\}$ is a real Cauchy sequence, as $0 \leqq h_{n}(T) \leqq 1 / 2 n$ for each $n$, and so for any $\varepsilon>0$ we can take an integer $p>1 / \varepsilon$, so that if $m, n \geqq p$, then $f_{m}, f_{n} \in F_{p}$, and hence $-2 h_{p}$ $<f_{m}-f_{n}<2 h_{p}$; thus $\left|f_{m}(T)-f_{n}(T)\right| \leqq 2 h_{p}(T)<\varepsilon$. 
Then $\mathscr{F}(T)$ is the limit of $\left\{f_{n}(T)\right\}$.

By standard methods it can be shown that $\mathscr{F}(T)$ is well defined, that $(-\mathscr{F})(T)$ $=-(\mathscr{F}(T))$ and $\mathscr{F}(T)+\mathscr{G}(T)=(\mathscr{F}+\mathscr{G})(T)$ for each $\mathscr{F}, \mathscr{G} \in A^{*}$, and, finally, that $\mathscr{V}(a)(T)=a(T)$ for each $a \in A$.

We then form $\psi: A^{*} \rightarrow B$, where $B$ is a group of real functions of $\mathscr{T}$, by the rule $\mathscr{F} \psi(T)=\mathscr{F}(T)$ for all $T \in \mathscr{T}$, for each $\mathscr{F} \in A^{*}$. We give the image the induced order.

If $\mathscr{F}>\mathscr{V}(0)$ in $A^{*}$, then we may choose our sequence $\left\{f_{n}\right\}$ entirely within $P$, the positive cone of $A$, as $P \in \mathscr{F}$; so $f_{n}(T) \geqq 0$ for each $n$ and each $T$, and hence $\mathscr{F}(T) \geqq 0$ for each $T \in \mathscr{T}$.

Suppose we have $\mathscr{W} \in A^{*}$ such that $\mathscr{W}(T) \geqq 0$ for all $T \in \mathscr{T}$. Take $\mathscr{F}>\mathscr{W}$ in $A^{*}$; as $A$ is a tight Riesz group, we have $u, v \in A$ such that $\mathscr{F}>\mathscr{V}(u)>\mathscr{V}(v)$ $>\mathscr{W}$. Hence $\mathscr{V}(v)(T) \geqq \mathscr{W}(T) \geqq 0$ for each $T \in \mathscr{T}$, by the preceding paragraph; and so $v(T) \geqq 0$ for each $T$, from which $v \geqslant 0$ follows as the representation of A is proper. Hence, as $u>v$, we have $u>0$, and so $\mathscr{F}>\mathscr{V}(0)$. Therefore $\mathscr{W} \succcurlyeq \mathscr{V}(0)$ in $A^{*}$.

Finally, $\psi$ is an $o$-isomorphism; for if we have $\mathscr{W} \psi=0$, i.e. $\mathscr{W}(T)=0$ for all $T \in \mathscr{T}$, then both $\mathscr{W} \succcurlyeq \mathscr{V}(0)$ and $-\mathscr{W} \succcurlyeq \mathscr{V}(0)$, that $\mathscr{W}=\mathscr{V}(0)$. Hence $\left(A^{*}, \geqq\right)$ is $o$-isomorphic to a properly $\beta$-ordered function group on $\mathscr{T}$; and $\mathscr{V}(a) \psi=a \phi$ for each $a \in A$.

Next we define $A_{T}=\{f(T) \mid f \in A\}$ and $A^{*}{ }_{T}=\left\{\mathscr{F}(T) \mid \mathscr{F} \in A^{*}\right\}$ for each $T \in \mathscr{T}$. Then we have

LEMMA 5.4. If $A_{T}$ is not a dense subgroup of the reals, then there is an $f>0$ in $A$ such that $f(T)=0$.

Proof: $h_{n}$ of theorem 5.3, for large enough $n$, must have $h_{n}(T)=0$.

Lemma 5.5. If there is an $f>0$ in $A$ with $f(T)=0$, then $A_{T}=A^{*}{ }_{T}$.

Proof: Take $\mathscr{F} \in A^{*}$; then there is an $F \in \mathscr{F}$ such that $F-F \subseteq(-f, f)$. If $g \in F$, then $F-g \subseteq(-f, f)$, so that $F \subseteq(g-f, g+f)$; thus $(g-f, g+f) \in \mathscr{F}$, i.e., $\mathscr{V}(g-f)<\mathscr{F}<\mathscr{V}(g+f)$. Thus

$$
g(T)=g(T)-f(T) \leqq \mathscr{F}(T) \leqq g(T)+f(T)=g(T),
$$

and so $g(T)=\mathscr{F}(T)$.

\section{6. o-simple Tight Riesz Groups}

In this section we will suppose $(A, \geqq)$ to be an isolated $o$-simple abelian tight Riesz group without pseudozeros; the aim of the section will be to extend a result of Fuchs' ((1965a), p. 198) to show that the completion $\left(A^{*}, \geqq\right)$ is in fact an $o$-simple tight Riesz vector space. Fuchs' proof may be easily adapted to obtain the result when $(A, \geqq)$ is divisible; hence it is necessary only to show that $\left(A^{*}, \geqq\right)$ 
is divisible, and Fuchs' result, so modified, can then be applied to $\left(A^{*}, \geqq\right)$, to obtain the result; for $\left(A^{* *}, \geqq\right)$ is 0 -isomorphic to $\left(A^{*}, \geqq\right)$ (Sherman (to appear) Thm. 2).

Lemma 6.1. For any $u<v$ in $A$ there is an $r \in A$ such that $2 r \in(u, v)$.

Proof: As $(A, \geqq)$ is directed we can find $z>u, 0$ in $A$, and so $2 z>u$; and, as $(A, \geqq)$ is $o$-simple and $y-u>0$, there is an integer $m$ such that $2^{m}(v-u)$ $>2 z-u$. Hence $u+2^{m}(v-u)>2 z>u$.

Put $y=u+2^{n-1}(v-u)$; we shall be using induction on $n$, from $m+1$ to 1 . Thus we suppose that $u+2^{n}(v-u)>2 w>u$ for some $w \in A$. Hence

$$
2 y-u=u+2(y-u)=u+2^{n}(v-u)>2 w>u,
$$

and $y>u$. Thus we have $y-w, w>u-y+w, u-w$. As $(A, \geqq)$ is a tight Riesz group, there is an $s \in A$ such that $y-w, w>s>u-y+w, u-w$; so $y-w, w>s, u-s$; hence there is a $t \in A$ such that $y-w, w>t>s, u-s$. So

i.e.,

$$
\begin{gathered}
y=y-w+w>t+t>u-s+s=u \\
u+2^{n-1}(v-u)>2 t>u .
\end{gathered}
$$

LEMMA 6.2. For any $u<v$ in $A$ and any integer $m$ there is $a w \in A$ such that $u<2^{m} w<v$.

ProOF. $(A, \geqq)$ is dense, so we can find $u_{0}, u_{1}, \cdots, u_{m} \in A$ such that $u=u_{0}$ $<u_{1}<u_{2} \cdots<u_{m}=v$. So by Lemma 6.1 we can find $z_{0}, z_{1}, \cdots, z_{m-1} \in A$ such that $u_{0}<2 z_{0}<u_{1}<2 z_{1}<\cdots<2 z_{m-1}<u_{m}$. As $(A, \geqq)$ is isolated, $z_{0}<z_{1}<\cdots$ $\cdots<z_{n-1}$; by induction, then, we find $u_{0}<2 z_{0}<4 t_{0}<\cdots<2^{m} w<\cdots<2 z_{m-1}$ $<u_{m}$. So $2^{m} w \in(u, v)$.

LEMMA 6.3 For any $v>u>0$ in $A$ there is an integer $m$ and $a y \in A$ such that both $2^{m} y$ and $\left(2^{m}+1\right) y \in(u, v)$.

Proof. Loy and Miller ((1972), page 236) showed that, for any $x>0$ in $A$ and any integer $n$, there is a $t>0$ in $A$ such that $x>n t$. Take $w \in A$ such that $w>0$ and $v-u>2 w$; so $u<u+w<u+2 w<v$.

As $(A, \geqq)$ is $o$-simple, there is an integer $m$ such that $2^{m} w>u+w$; by lemma 6.2 we can find $y \in A$ such that $u+w>2^{m} y>u>o$; so $2^{m} w>2^{m} y>0$. As $(A, \geqq)$ is isolated, then $w>y>0$. Hence $v>u+2 w>2^{m} y+y>u$. Thus $v>\left(2^{m}+1\right) y>2^{m} y>u$.

Lemma 6.4. For any $u<v$ in $A$ an any integer $n$ there is a $z \in A$ such that $n z \in(u, v)$.

PROOF: If $u>0$, take $p$ an integer such that $2^{p}>n$, and let $w$ be such that $u<w<v$. By lemma 6.2, we find $y, t \in A$ such that $2^{p} y \in(u, w)$ and $2^{p} t \in(w, v)$; so $0<y<t$, by the isolation of $(A, \geqq)$, and so, by lemma 6.3 we have $s \in A$ 
and an integer $m$ such that $2^{m} s$ and $\left(2^{m}+1\right) s \in(y, t)$. So $s>0$, and $2^{m+p} s$, $\left(2^{m+p}+1\right) s, \cdots,\left(2^{m+p}+2^{p}\right) s \in(u, v)$; but there are more that $n$ consecutive integers from $2^{m+p}$ to $2^{m+p}+2^{p}$, and so one of them must be of the form $n k$, where $k$ is an integer. Putting $z=k s$, then, we have $n z=n k s \in(u, v)$.

If $u \ngtr 0$, then choose $x, y \in A$ such that $0<x<v-u$ and $y<u, 0$; thus $y-x<y$ and $u-y+x<v-y$. Hence $(y-x, y)+(u-y+x, v-y)=(u, v)$, as $(A, \geqq)$ is a tight Riesz group (cf. Loy and Miller (1972), p. 228), whilst $y<0$ and $u-y+x>0$. Thus by the previous section we can find $s, t \in A$ such that $n s \in(-y, x-y)$ and $n t \in(u-y+x, v-y)$; so $n(t-s) \in(u, v)$.

THEOREM 6.5. The completion $\left(A^{*}, \geqq\right)$ is divisible.

Proof: Let $n$ be an integer; then for any round Cauchy $O$-filter $\mathscr{F} \in A^{*}$ we form the filter base $\{(s, t) \mid(n s, n t) \in \mathscr{F}\}$, and from this we generate an $O$-filter $\mathscr{G}$. From Sherman (to appear) we know that for any $(a, d) \in \mathscr{F}$ there is a $(b, c) \in \mathscr{F}$ such that $a<b<c<d$; so, by lemma 6.4 we can find $s<t$ in $A$ such that $a<n s<b<c<n t<d$; and so $(s, t) \in \mathscr{G}$. It is then easily verified that $G \in A^{*}$, and $n \mathscr{G}=\mathscr{F}$.

Corollary 1. $\left(A^{*}, \geqq\right)$ is an o-simple tight Riesz (real) vector space.

Proof: It remains only to show that $\left(A^{*}, \geqq\right)$ is $o$-simple. Given $\mathscr{F}>\mathscr{V}(0)$ in $A^{*}$, and $\mathscr{G} \in A^{*}$, we take $f, g \in A$ such that $\mathscr{F}>\mathscr{V}(f)>\mathscr{V}(0)$ and $\mathscr{V}(g)>\mathscr{G}$; as $(A, \geqq)$ is $o$-simple, there is an integer $n$ such that $n f>g$; so $n \mathscr{F}>\mathscr{G}$.

Loy and Miller ((1972), p 236) and Reilly (1971) have shown that tight Riesz groups with lattice ordered associated order are isolated, and hence so are any subgroups of them; thus, by theorem 6 of Sherman (to appear), which states that approximation antilattices are dense subgroups of such groups, and so are isolated, we can extend Fuchs' result to

COROLLARY 2. If $(A, \geqq)$ is an o-simple approximation antilattice, then $\left(A^{*}, \succcurlyeq\right)$, its Fuchs completion, is a real vector lattice.

\section{Examples}

Example 7.1. Consider $A=R^{2}$ with the positive cone $P=\{(x, y) \mid x>0$ and $y>0$; or $x=0, y>0$ and $y \in Q\}$, where $Q$ is the set of rationals. Then $\{(x, y) \mid x=0, y \in Q\}$ is the minimal non-zero $o$-ideal; we shall denote it by $\{0\} \times Q$, and its co.mpletion we can denote by $R$. Then theorem 3.3 gives us

$$
A^{*} \cong(R \times R) \times R /\{(0, q,-q) \mid q \in Q\}
$$

Note that, in reference to theoren 5.3, $A$ is a $\beta$-ordered function group on a set $\mathscr{T}$ of two elements, but not a proper one; and $A^{*}$ is not $o$-isomorphic to any function group on $\mathscr{T}$.

EXAMPLE 7.2. Consider as $A$ the group of functions from $N$, the set of positive 
integers to $Z$, the group of integers, lexicographically ordered; i.e., $f>0$ in $A$ if there is an $n \in N$ such that $f(n)>0$ and $f(m)=0$ for all $m<n$ in $N$. As each $Z$ is a Riesz group, and the index set has no last element, $A$ must be a tight Riesz group. Further, it is clear that each interval of the form $(-f, f)$ around 0 contains the $o$-ideal $B_{n}=\{g \in A \mid g(m)=0$ for all $m \leqq n\}$, where $f(n)$ is the first nonzero component of $f$. Thus theorem 4.1 applies to $A$.

Clearly all the $o$-ideals are of the form of the one given above, and so their factor groups are all of the form $A_{n}=\left\{g+B_{n} \mid g(m)=0\right.$ for all $\left.m>n\right\}$. Thus from theorem 4.1 it can readily be seen that $A^{*}$ will be $o$-isomorphic to $A$.

EXAMPLE 7.3. The following example is of an $\alpha$-ordered function group, whose completion, considered as a function group on the same set, is not $\alpha$-ordered. Thus, in theorem 5.3 " $\beta$-ordered" may not be replaced by " $\alpha$-ordered".

Consider $A$ to be that subgroup of $C(0,1)$ consisting of all those functions whose graphs are made up of a finite number of straight line segments; we put $f>0$ in $A$ if $f(x)>0$ for all $x \in(0,1)$. In view of the usual methods of approximating uniformly continuous functions with functions of this kind, it is clear that $A^{*}$ is $o$-isomorphic to the group of uniformly continuous functions on $(0,1)$.

It is easily verified that $(A, \geqq)$ is a tight Riesz group without pseudozeros, and it is clearly also an $\alpha$-ordered function group on $(0,1)$.

If $\left(A^{*}, \geqq\right)$ is also to be an $\alpha$-ordered function group on $(0,1)$, then we would have to have $g:(0,1) \rightarrow R, x \rightarrow x^{2}$ a positive function; as $(A, \geqq)$ is a tight Riesz group, we should then be able to find an $f>0$ in $A$ such that $g>f$; but this would mean that the first segment of $f$ would have to be along the 0 -line, contradicting $f>0$. Hence $\left(A^{*}, \geqq\right)$ cannot be $\alpha$-ordered.

EXAMPLE 7.4. In section 6 it is natural to wonder whether any of the conditions may be relaxed. It may be true that any $o$-simple abelian tight Riesz group without pseudozeros is isolated; I have neither proven nor disproven it. However, the following two examples are of an $o$-simple abelian $T R(1,2)$ group without pseudozeros which is not isolated, and an abelian tight Riesz group without pseudozeros which is not $o$-simple and also not isolated.

Consider $A$ to be $R^{2}$ again, with positive cone $P=\{(x, y) \mid x>0, y>0$, and for each positive integer $n$ for which $x+y \leqq 2 n$, we have $x-y>2 n-2\}$; this is the set in the first quadrant of the plane bounded by, but not including, the $y$-axis and a sawtooth curve starting from $(0,0)$, going straight to $(1,1)$, then to $(2,0)$, and back up to $(3,1)$, etc.

In this case $(A, \geqq)$ is not isolated, but is an $o$-simple $T R(1,2)$ group without pseudozeros. In fact $A^{*}$ is $o$-isomorphic to $A$, so that the conclusion of theorem 6.5 corollary 1 does not hold.

EXAmPLE 7.5. This is a non-isolated tight Riesz group, and is very similar to example 7.1. Consider $R^{2}$ with a positive cone $P=\{(x, y) \mid x>0, y>0$; or 
$x=0$, and $y=m / 2^{n}$, where $m$ and $n$ are positive integers $\}$. Clearly $(0,1)>(0,0)$ but $\left(0, \frac{1}{3}\right)$ is not, so that $A$ is not isolated; and $A$ can be shown to be a tight Riesz group without pseudozeros.

EXAMPLE 7.6. Consider the group $A$ of bounded rational-valued functions on $\mathscr{T}=[0,1]$, with the order $f>0$ if $f(T) \geqq 0$ for all $T \in \mathscr{T}$, as long as $f(T)=0$ for only finitely many $T$. Then $A$ is a tight Riesz group without pseudozeros, and by theorem 5.3 and lemma $5.5, A^{*}$ is $o$-isomorphic to $A$.

EXAMPLE 7.7. It is possible for a p.o. group to have a bounded subgroup, but no bounded $o$-ideal and no pseudozeros, as the following example shows:

Consider $A$ to be the direct product of $Z$ with the group $B$ of bounded real functions on $R$, and order it by putting $(f, n)>(0,0)$ if (i) if $n=0$, then $f(x) \geqq 0$ for all $x \in R$, where $f(x)=0$ for only finitely many $x$; or (ii) if $n \neq 0$, then $f(x)>0$ for all $x \in R$.

Clearly $\{(0, n) \mid n \in Z\}$ is a bounded subgroup, but, as any $o$-ideal contains a positive element, there is no bounded $o$-ideal. $(A, \geqq)$ has no pseudozeros; the only possible pseudozeros are those elements of the form $(0, n)$ where $n \neq 0$; but if we take $(f, 0)$ where $f(x)>0$ for all $x$ except $\frac{1}{2}$, and $f\left(\frac{1}{2}\right)=0$, then $(f, 0)>(0,0)$, but $(f, 0) \ngtr(0, n)$. Further, $(A, \geqq)$ is a $T R(1,2)$ group but not a tight Riesz group.

\section{References}

J. Dieudonné (1941), 'Sur la Théorie de la Divisibilité,' Bull. Soc. Math. Fr. 69, 133-144.

L. Fuchs (1963), Partially Ordered Algebraic Systems, (Pergamon Press, 1963).

L. Fuchs (1965), 'Riesz Groups,' Ann. Scuela Norm. Sup. Pisa (3) 19, 1-34.

L. Fuchs (1965a), 'Approximation of Lattice Ordered Groups', Ann. Univ. Sci. Budapest Eötvös, Sect. Math. 8 187-203.

G. Grätzer (1968), Universal Algebra, (Van Nostrand, 1968).

P. Lorenzen (1939), 'Abstrakte Begründung der Multiplikativen Ideal theorie', Math. Zeit 45 433-553.

R. Loy and J. Miller (1972), 'Tight Riesz Groups,' J. Austral. Math. Soc. 13 (2) (1972), 224-40.

N. Reilly (1971), Compatible Tight Riesz Orders and Prime Subgroups. (Preprint, Simon Fraser University, 1971).

N. Reilly (1972), 'Permutational Products of Lattice Ordered Groups', J. Math. Austral. Soc. 13 25-34.

P. Ribenboim (1959), Théorie des Groups Ordonnés, (Bahia Blanca, 1959.)

H. Schaefer (1966), Topological Vector Spaces, (Springer-Verlag, 1966.)

B. Sherman 'Cauchy Completion of Partially Ordered Groups'. J. Austral. Math. Soc. (to appear).

Department of Mathematics

Monash University

Clayton, 3168

Australia 